\title{
Die elektrische Erregbarkeit des menschlichen Auges.
}

\author{
Von \\ Kituya Iwama. \\ (岩間 吉也)
}

(Aus dem II. Institut für Physiologie der Tohoku-Universität

zu Sendai. Vorstand: Prof. Dr. K. Motokawa.)

(Received for publication, December 7, 1944)

\section{Einleitung.}

Die sinnesphysiologischen Studien am menschlichen Auge werden im allgemeinen mit dem Lichtreiz, dem einzigen adäquaten Reiz für das Auge ausgeführt. Aber, wenn wir das Auge gleich den Nerven oder Muskeln als reizbares Gebilde auffassen, ist die elektrische Reizung die beste Untersuchungsmethode, denn wir können dank der Genauigkeit und Einfachheit dieses Verfahrens damit eingehende Untersuchungen durchführen.

Es ist seit langem bekannt, dass man einen kurzdauernden Lichtblitz-Phosphen-empfindet, wenn das Auge elektrisch gereizt wird. Wird diese Erscheinung als Merkmal der elektrischen Erregbarkeit des Auges gewählt, so kann man das menschliche Auge nicht nur sinnes-, sondern auch reizphysiologisch untersuchen.

Um die physiologische Bedeutung der elektrischen Reizung des Auges zu klären, muss man in erster Linie die Beziehung zwischen den optischen und den elektrischen Reizgesetzen kennen.

- In vorliegender Arbeit habe ich folgende Fragen zu lösen versucht:

1. Wie ist die Reizzeit-Spannungsrelation beim Auge? und

2. wie wird die elektrische Erregbarkeit des Auges durch die Dunkeladaptation beeinflusst?

Die Reizzeit-Spannungsrelation ist eine grundlegende Beziehung in der Reizphysiologie; Chronaxie und Rheobase, die sich aus der ReizzeitSpannungsrelation bestimmen lassen, geben uns genaue Kenntnis von der elektrischen Erregbarkeit des betreffenden Gebildes.

\section{Versuchsmethode.}

Das Schaltungsschema für die Reizung mit rechteckigen Stromstössen 
ist links oben in Abb. 1 dargestellt. Durch die Abstufung des Widerstandes $\mathrm{R}$ wurden Gleichspannungen beliebiger Grösse hergestellt. Die Zeitdauer der Stromstösse wurde mittels Hashidaschen Rheotoms abgestuft. Um Veränderungen des Kopfwiderstandes vorzubeugen, wurde ein induktionsfreier Widerstand von $10 \mathrm{~K} \Omega$ dem Kopf vorgeschaltet. Der Strom wurde mittels einer auf die Stirn gesetzten differenten und einer auf das Hinterhaupt gelegten indifferenten Elektrode dem Kopf der Versuchsperson zugeführt. Die Elektroden bestanden aus chrolierten Silberplatten von $2 \times 3 \mathrm{qcm}$ und wurden mittels Paste und Heftpflasters auf den Kopf fixiert.

Ich untersuchte die elektrischen Schwellenwerte, indem ich die beiden

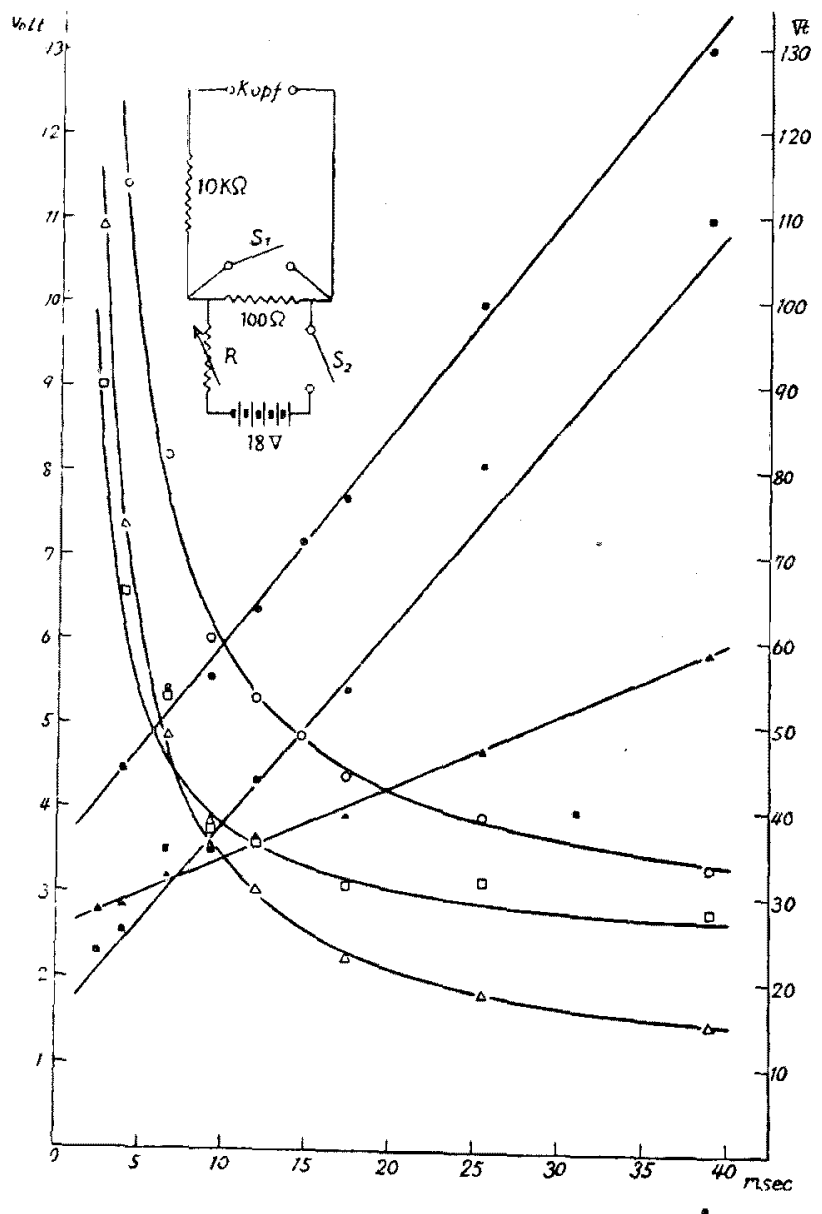

Abb. 1. Zeitspannungskurve unter verschiedenen Adaptationszuständen. $\square$ : Bei Helladaptation (Beleuchtung: 950 lux), $\triangle$ : Bei mittelmässiger Adaptation, (Beleuchtung: 3 lux), $\bigcirc$ : Bei Dunkeladaptation (Beleuchtung: 0). Die Quantitätskurven sind durch dementsprechende gefülte Marke gekennzeichnet. 
Elektroden auf verschiedene Stellen des Kopfes aufsetzte, und stellte fest, dass der Schwellenwert niedrig ist, wenn wenigstens ein Elektrode in der Nähe des Auges liegt, und dass er am niedrigsten ist, wenn man die Elektroden in oben erwähnter Weise, nämlich, die eine auf der Stirn und die andere auf dem Hinterhaupt anbringt. Aus diesen Gründen benutzte ich bei den vorliegenden Versuchen die oben erwähnte Elektrodenanordnung.

\section{Versuchsergebnisse.}

1. Reizzeit-Spannungsrelation bei verschiedenen Adaptationszuständen.

Ohne Rücksicht auf den Adaptationszustand hat eine Anzahl von Forschern die Reizzeit-Spannungsrelation des Auges untersucht und festgestellt, dass das W e is s sche Gesetz bei Gleichstromreizung ( $\mathrm{Sch}$ ri e ve $\left.{ }^{11}\right)$ oder das H o or w e g sche Gesetz bei Reizung mittels Kondensatorentladung (Bourguignon u. Déj e a n, ${ }^{2)} \mathrm{Sch}$ riever ${ }^{1)}$ ) gültig ist. Achelis u. Merkulow haben mittels Kondensatorentladung die Gültigkeit des Hoorweg schen Gesetzes unter verschiedenen Adaptationszuständen untersucht, aber ihre Resultate erwiesen sich als nicht eindeutig und ihre Deutung war zum Teil irrtümlich.

Bei meinen Versuchen benutzte ich zur Herstellung der Helladaptation ein Adaptationsgerät mit gleichmässiger Wandbeleuchtung von 950 lux. Um die Augen in einem mittelmässigen Adaptationszustand zu erhalten, sass die Versuchsperson in einem halbverdunkelten Zimmer und schaute in den Schatten des eigenen Kopfes, wobei eine Lampe von 40 Watt hinter dem Kopf angebracht war. Die Beleuchtung im Schatten betrug etwa 3 lux. Die Untersuchung bei Dunkeladaptation wurde erst nach 40 Minuten langem Aufenthalt der Versuchsperson in völlig dunklem Raum ausgeführt.

Die unter den oben erwähnten dreierlei Versuchsbedingungen erhaltenen Resultate sind in Abb. 1 graphisch dargestellt. Die Quantitätskurven stehen, wie die ausgezogenen Geraden zeigen, mit der Zeit befriedigend in linearem Zusammenhang, was darauf hinweist, dass das Weisssche Gesetz unter den verschiedenen Adaptationszuständen gültig ist, und dass jede experimentell bestimmte Zeitspannungskurve mit einer einzigen Hyperbel dargestellt werden kann. Aber die Reizzeit-Spannungskurve konnte unter Umständen nicht ganz stetig verlaufen, so dass sie aus beiden Hyperbeln zusammengesetzt zu sein schien. Dasselbe Verhältnis ist schon von Achelis u. Merkulow beobachtet worden. Diese Autoren wollten diese Zweiteiligkeit der Kurve auf die zweierlei reizbaren Elemente der Netzhaut, die Stäbchen und Zapfen zurückführen. Aber diese Deutung erweist sich ohne weiteres als unrichtig, wenn 
Art und Weise der Kreuzung der beiden Kurventeile genauer beobachtet werden; die extrapolierte Strecke des einen Kurventeils (gestrichelt in Abb. 2) verläuft unter dem anderen Kurventeil, so dass die Schwelle dieser fiktiven Kurvenstrecke niedriger als die experimentelle sein muss. Es ist ganz unbegreiflich, warum der wirkliche Kurventeil nicht durch diese niedrigere fiktive Schwelle sondern durch die höhere bestimmt wurde. Die Zweiteiligkeit muss daher auf andere Ursachen zurückgeführt werden.

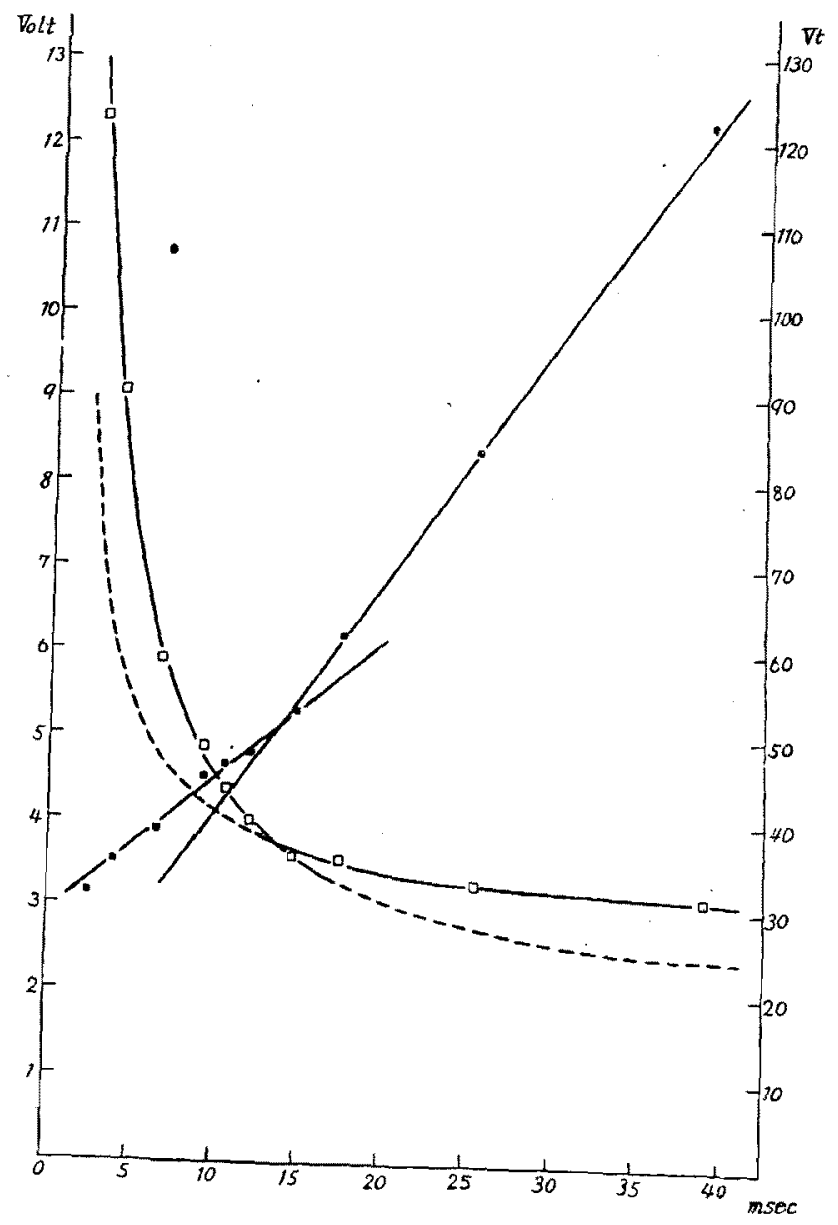

Abb. 2. Vom Weiss schen Gesetz abweichendes Beispiel.

Zur Erklärung dieser Kurvenzweiteiligkeit muss man vor allem die Polarisationskapazität der Haut in Betracht ziehen. Wenn man dem Gewebe eine konstante Spannung anlegt und den Strom durch die Haut hindurch fliessen lässt, so wird die Stromstärke durch die Polarisationskapazität der Haut innerhalb einiger Millisekunden stark herabgesetzt, so dass Reize von langer Reizzeit verhältnismässig wenig wirksam sind. Unter diesen Bedingungn muss man die Reizspannung erhöhen, und 
somit muss die Quantitätskurve für längere Reizzeiten steiler verlaufen als die für kürzere. Auf diese Weise kommt die Zweiteiligkeit der Kurve zustande.

Diese Erklärung scheint aber dem Umstand zu widersprechen, dass sich die Zeitspannungskurve in Abb. 1 innerhalb der Fehlergrenze nur mit einer einzigen Hyperbel darstellen lässt, oder dass die Quantität mit der Zeit linear zusammenhängt. Dabei handelt es sich um den besonderen Verlauf des Stromes, der durch die Polarisation innerhalb einiger Millisekunden stark herabgesetzt wird und ein Minimum erreicht, nimmt allmählich mit der Zeit wieder zu, wie ich mittels eines elektromagnetischen Oszillographen wirklich bestätigen konnte. Dank dieser Erhöhung der Stromstärke wird die Schwelle für lange Reizzeiten verhältnismässig niedrig bleiben, so dass die Quantitätskurve für längere Reizzeiten nicht immer steiler als die für kürzere verläuft.

Der bei dieser Untersuchung erhaltene Chronaxiewert beträgt bei mittellmässiger Adaptation 30, $8 \mathrm{msec}$, bei Helladaptation 6,2 msec und bei Dunkeladaptation $13,8 \mathrm{msec}$. Wie mehrere Forscher miteinander übereinstimmend bemerken, sind die Chronaxiewerte des Auges viel grösser als die der Muskeln oder Nerven. So grosse Werte kann man freilich nicht dem Sehnerven zuschreiben. Es scheint mir, dass die Ganglienzellen der Netzhaut der Angriffspunkt des elektrischen Reizes sind, denn Ganglienzellen besitzen im allgemeinen eine klejnere Reaktionsgeschwindigkeit und zeigen somit längere Chronaxiewerte als periphere Nerven. Es ist hervorzuheben, dass die Chronaxie bei der mittelmässigen Adaptation am grössten ist, und dass die Rheobase am kleinsten ist.

2. Veränderungen der elektrischen Schwellenwerte während des Dunkeladaptationsverlaufes.

Die allgemein bekannte Kurve des Dunkeladaptationsverlaufes erhält man mittels des $\mathrm{Nagelschen} \mathrm{Adaptometers.} \mathrm{Anstatt} \mathrm{des} \mathrm{Lichtreizes}$ können wir durch den elektrischen Reiz die zeitlichen Veränderungen der Schwellenwerte verfolgen. In Abb. 3 sind die Ergebnisse für Reize von verschiedener Dauer abgebildet. Wie aus dieser Abb. hervorgeht, ist die Veränderung der Schwellenwerte sehr regelmässig, und unabhängig von der Reizdauer lässt sich folgende Gesetzmässigkeit ableiten; direkt nach dem Ausschalten des helladaptierenden Lichtes ist der Schwellenwert am niedrigsten, welcher allmählich mit dem Fortschreiten der Adaptation zunimmt und schliesslich einen bestimmten Endwert erreicht. Aber es ist bemerkenswert, dass der Verlauf dieser Kurve je nach der angewandten Reizdauer voneinander ziemlich verschieden sind: 1. Die Kurven für kürzere Reizzeiten liegen im allgemeinen höher als die für längere, und 2. die Kurven erreichen ihren eigenen Endwert bei kürzeren Reizzeiten 


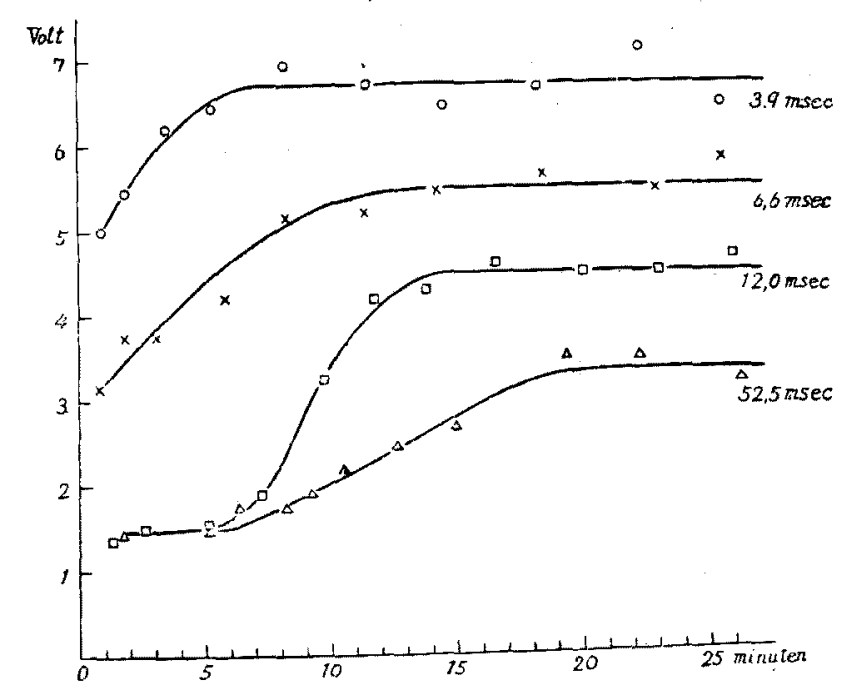

Abb. 3. Zeitliche Veränderungen der Schwellenwerte während der Dunkeladaptation. Die Dauer des Reizstromes ist jeder Kurve beigegeben.

viel schneller als bei längeren. Wie aus $\mathrm{Abb}$. 3 ersichtlich, erreicht der Schwellenwert für die Reizzeit 3,9 msec schon 10 Minuten nach dem Beginn der Dunkeladaptation seinen Endwert, während der für die Reizzeit 12,0 msec bei gleicher Dunkeladaptationszeit nur zwei Drittel seines Endwertes beträgt.

Durch die Dunkeladaptation wird die Erregbarkeit des Auges für das Licht allmählich erhöht, und man braucht mehr als 40 Minuten, um die höchste Erregbarkeit zu erreichen. Aber bei elektrischer Reizung wird der Endwert der Schwelle selbst für eine hinreichend lange Reizdauer (in meinem Experiment 52,5 msec) in etwa 20 Minuten erreicht. Aus diesem Ergebnis ergibt sich ohne weiteres, dass die Erregbarkeit der Ganglienzellen während der Dunkeladaptation in ganz anderer Weise als die lichtempfindlichen Zellen der Netzhaut verändert wird.

\section{Diskussion.}

Wie oben ausgeführt, verändert sich die elektrische Erregbarkeit des Auges während der Dunkeladaptation. Nun erhebt sich die Frage, wie Chronaxje und Rheobase während dieser Zeit verändert werden. Um die Frage beantworten zu können, muss man die Reizzeit-Spannungsrelation für jeden Augenblick der Dunkeladaptation kennen lernen, was aber in üblicher Weise kaum möglich ist. Ich konnte aus dem Versuchsergbnis 2 die Zeitspannungskurve für jeden Zeitpunkt der Dunkeladaptation in folgender Weise konstruieren: Aus der Kurvenschar in Abb. 3 erhält man eine Reihe der Schwellenwerte für ein und denselben Zeitpunkt. Trägt man die so erhaltenen Werte als Ordinaten und die dementsprechenden 
Reizzeiten als Abzissen auf, so kann man die Reizzeit-Spannungskurve für den in Frage TABELLE I. stehenden Zeitpunkt erhalten. Auf diese Weise gen der Chronaxie und wurden die Kurven für etwa zehn voneinander Rheobase während der verschiedene Zeitpunkte während der Dunkel- Dunkeladaptation. adaptation ermittelt. Die Chronaxiewerte, welche aus den so konstruierten Kurven erhalten wurden, sind in Tabelle I wiedergegeben. Die Chronaxie steigt, wie aus der Tabelle hervorgeht, mit dem Fortschreiten der Dunkeladaptation langsam an, erreicht nach 10-12 Minuten ein Maximum und sinkt darauf allmählich, um schliesslich in etwa 20 Minuten zu einem kons tanten Wert gelangen.

Es ist von Interesse, dass der Chronaxiewert auf diese Weise im Adaptationsverlauf ein Maximum passiert, was mit dem oben darglegten Ergebnis übereinstimmt, dass der Chronaxiewert bei mittelmässiger Adaptation viel grösser als der bei Hell- und Dunkeladaptation ist.

\begin{tabular}{c|c|c}
\hline & $\begin{array}{c}\text { Chronaxie } \\
(\mathrm{msec})\end{array}$ & $\begin{array}{c}\text { Rheobase } \\
\text { (Volt) }\end{array}$ \\
\hline Hell & 8,3 & 3,4 \\
\hline Dunkel & & \\
$2^{\prime}$ & 7,75 & 1,35 \\
$5^{\prime}$ & 8,5 & 1,5 \\
$8^{\prime}$ & 9,5 & 1,7 \\
$10^{\prime}$ & 10,3 & 2,0 \\
$12^{\prime}$ & 11,2 & 2,25 \\
$15^{\prime}$ & 6,5 & 2,75 \\
$17^{\prime}$ & 4,9 & 3,1 \\
$20^{\prime}$ & 3,5 & 3,4 \\
$22^{\prime}$ & 3,5 & 3,45 \\
$25^{\prime}$ & 3,5 & 3,45 \\
& &
\end{tabular}

\section{Zusammenfassung.}

1. Die Reizzeit-Spannungsrelation des menschlichen Auges wurde unter verschiedenen Adaptationszuständen untersucht. Die Chronaxie betrug bei mittelmässiger Adaptation $30,8 \mathrm{msec}$, bei Helladaptation 6,2 msec und bei Dunkeladaptation 13,8 msec.

2. Die Schwelle für rechteckige Stromstösse von verschiedener Dauer wurde während des Dunkeladaptationsverlaufs verfolgt. Die Schwelle war direkt nach dem Ausschalten des helladaptierenden Lichtes am niedrigsten, stieg mit der Zeit allmählich an und erreichte einen konstanten Wert. Diese Veränderung ging um so schneller vor sich, je kürzer die Reizzeit war.

3. Während der Dunkeladaptation passiert die Chronaxie ein Maximụm.

\section{Literatur.}

(1) Schriever, H.; Z. Biol., 1930, 90, 347.

(2) Bourguignon, G. u. Déjean, R.; C. r. S. Biol., 1926, 94, 750.

(3) Achelis, J. D. u. Merkulow, J.; Z. für Sinnesphysiol., 1930, 60, 95. 